\title{
Histamine-releasing factor: a promising therapeutic target for food allergy
}

\begin{abstract}
Marsha Wills-Karp
Department of Environmental Health and Engineering, Johns Hopkins Bloomberg School of Public Health, Baltimore, Maryland, USA.
\end{abstract}

\begin{abstract}
The prevalence of food allergies has been increasing at an alarming rate over the last few decades. Despite the dramatic increase in disease prevalence, the development of effective therapies has not kept pace. In this issue of the $J C I$, Ando et al. provide a causal link between histamine-releasing factor (HRF) interactions with IgE and food allergy in a murine model. Successful oral immunotherapy of both egg-allergic human patients and food-allergic mice was associated with sustained suppression of HRF-reactive IgE levels. These results support a role for HRF-IgE interactions in the amplification of intestinal inflammation and suggest HRF as a therapeutic target in food allergy.
\end{abstract}

ety of fundamental cellular functions, such as cell-cycle progression, proliferation, and malignant transformation (10). HRF is also secreted by many cell types, and extracellular HRF has cytokine-like functions, including stimulation of histamine release and type 2 cytokine production from IgE-sensitized basophils and mast cells (11). Since its first description, HRF has been identified in nasal lavages, bronchoalveolar lavages, and skin blister fluids of allergic individuals (12-14). Several studies have shown that decreases in HRF levels track with a reduction of allergic symptoms in those patients undergoing successful immunotherapy, while HRF levels remain steady in those individuals that are nonresponsive to immunotherapy $(15,16)$. Collectively, these studies provide strong support for a role for $\mathrm{HRF}$ in allergic pathogenesis.

Although the contribution of HRF in the allergic diathesis has been studied for more than 30 years, efforts to establish a definitive role for this protein in allergic diseases had been hampered by the lack of an animal model in which to explore in vivo HRF functions, as HRF-knockout mice are embryonically lethal (10). However, mice with overexpression of HRF specifically in club cells exhibit an enhanced allergic phenotype after antigen sensitization and challenge, concomitant with elevated IL-4 and eosinophil levels when compared with littermate controls (17). While supportive of a role for HRF in allergy, these studies did not allow dissection of HRF's extracellular and intracellular actions.

Another impediment to defining HRF function in allergy has been a lack of understanding of its mode of action and the nature of its receptor. While early studies hinted that HRF might work either in concert with IgE or through IgE-dependent mechanisms, the fact that HRF activates some cells that do not express Fc receptors and that HRF does not interact with all IgEs (18) complicated understanding the role of HRF in the context of allergic inflammation. In 2012, Kashiwakura and colleagues (19) reported that HRF bound 


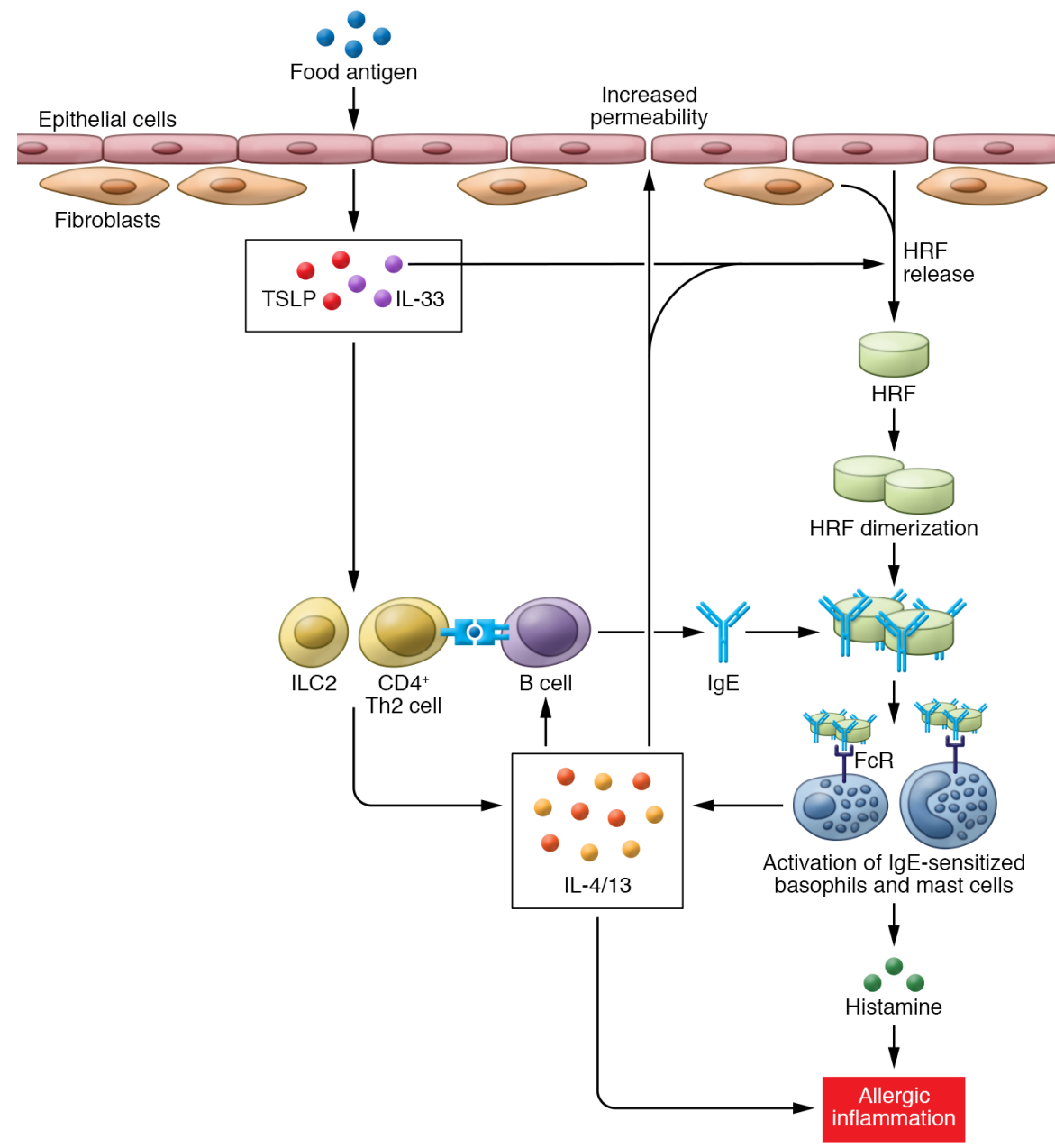

Figure 1. HRF amplifies the allergic response. Exposure to food allergen results in release of inflammatory cytokines, including IL-33 and TSLP, from the epithelium, which in turn promotes the development of a Th2 inflammatory response that results in release of IL- 4 and IL- 13 and the development of food allergen-specific IgE. IL-4, IL-13, and IL-33 induce secretion of HRF from the intestinal epithelium and underlying fibroblasts. HRF dimerization is thought to crosslink IgE and enhance binding and activation of mast cells and basophils via FcRI. Activation of these cells results in the release of histamine, IL-4, and IL-13, thereby resulting in allergic inflammation, such as is observed in food allergy, asthma, and allergic rhinitis. Additionally, release of IL-4 and IL-13 from mast cells and basophils further exacerbates the allergic response by maintaining HRF secretion.

to a subset of $\operatorname{IgE}$ and $\operatorname{IgG}$ antibodies by interacting with the Fab region (antigen binding) and not the Fc (FcR-binding region) regions of immunoglobulins, thereby establishing immunoglobulins as receptors for HRF. As previously reported (18), Kashiwakura et al. showed that not all IgE or Ig molecules bound HRF. Those Igs that did bind HRF were designated as HRF-reactive Igs, though it is not exactly clear what distinguishes HRF-reactive Igs from non-HRF-reactive Igs, and this area requires further study.

In the same report, Kashiwakura and colleagues (19) identified two distinct Ig-binding regions within $\mathrm{HRF}$ - one in the
$\mathrm{N}$ terminus (N-19) and the other in internal regions (H3 residues 107-135) of HRF. The discovery of these Ig-binding sites in HRF opened up the possibility of developing competitive inhibitors of HRF-IgE interactions. Indeed, tagging both $\mathrm{N} 19$ and $\mathrm{H} 3$ peptides with glutathione S-transferase (GST) (GST-N19 and GST-H3) inhibited the interaction between HRF and HRFreactive Abs. As they showed that HRF exists as a dimer, they theorized that the HRF dimer binds to two molecules of IgE and that this crosslinking of HRF to reactive IgE in turn promotes binding to Fc\&R1s on cells, such as mast cells and basophils, thereby leading to mediator release. They went on to show that pretreatment of mice in vivo with either of the HRF peptides (GST-N19, GST-H3) abrogated passive cutaneous anaphylaxis (PCA) induced by intradermal antigen challenge of mice primed with HRF-reactive IgE. As combining the two reagents (N19, H3) largely ameliorated the PCA reactions, there is clearly a dose-dependent effect. Taken together, these studies definitively established a link between HRF and allergic inflammation in vivo and suggested that HRF's role was downstream of IgE/FcR interactions, not upstream.

\section{HRF inhibition reduces allergy symptoms}

In this issue, Ando et al. (3) demonstrated that treatment of food-sensitized mice with the HRF competitive inhibitor GST-N19 prior to food challenge reduced diarrhea occurrence, concomitant with reductions in mast cell numbers, mast cell protease levels, and type 2 cytokines. HRF levels were 3 times higher in food-allergic mice than in controls and coincided with an increased HRF multimer-to-monomer ratio. HRF inhibitors had no effect on food allergy-associated symptoms in FceR1a-deficient mice; however, HRF inhibition suppressed allergic symptoms in FcrRIIB-deficient mice, suggesting that the downstream effects of HRF-IgE interactions are mainly mediated via FceR1. After food allergy was established, Ando and colleagues noted that therapeutic HRF inhibition did not reduce IgE levels, but did inhibit mast cell activation and reduce clinical symptoms. These findings suggest that HRF regulates the level of allergic inflammation, but is not able to reverse antigen sensitization once it has occurred. Nonetheless, these studies support the possibility that therapeutic administration of HRF inhibitors may be effective at suppressing the severity of food allergy.

Although not a lot is known about how the release of HRF is regulated during allergic inflammation, Ando and colleagues (3) showed that the Th2 cytokines (IL-4, IL-5, IL-13) induce HRF release from intestinal epithelial cells and fibroblasts and that epithelial-derived IL-33 enhances HRF secretion from fibroblasts. Based on their collective results, Ando et al. proposed a scenario in which HRF amplifies or perpet- 
uates allergic inflammation triggered by allergen-driven activation of $\mathrm{CD} 4^{+} \mathrm{T}$ cells, type 2 innate lymphoid cells (ILC2s), and type 2 cytokine (IL-13, IL-4) production, which in turn coordinate a series of events leading to IgE-dependent mast cell and basophil activation (Figure 1).

Furthermore, Ando et al. examined the association between HRF-reactive IgE levels and food allergy in patients before and after undergoing rush oral immunotherapy to assess the importance of HRF-reactive IgE levels in human food allergy (3). HRF-reactive IgE levels were higher in egg-allergic patients than in normal healthy controls. Moreover, following a period of allergen avoidance after the completion of oral immunotherapy, HRF-reactive IgE levels were reduced in patients in whom oral immunotherapy successfully reduced sensitivity to egg protein. Interestingly, having high HRF-reactive IgE levels before oral immunotherapy initiation was predictive of oral immunotherapy success, suggesting that symptoms may be highly dependent upon HRF-mediated inflammation. These studies suggest that serum HRF levels may be a good predictor of successful immunotherapy in food-allergic patients. These conclusions are supported by reports of an association between HRF levels and clinical status of food allergic patients (5).

Similar effects were observed in a mouse model of oral immunotherapy. Specifically, oral OVA desensitization prevented subsequent OVA challengeinduced diarrhea in OVA-sensitized mice and this benefit was concomitant with a marked reduction in HRF-reactive $\operatorname{IgE}$ levels (3). In a therapeutic oral immunotherapy model, which is more reflective of the human situation, mice rendered allergic by sensitization prior to the desensitization protocol had lower levels of HRF-reactive IgE, but no changes in OVA-specific IgE levels. The oral immunotherapy-tolerized mice also exhibited a reduction in the frequency of diarrheal occurrences following an oral OVA challenge when compared with nontolerlized mice. Based on these results, Ando and colleagues concluded that a reduction in HRF-reactive IgE levels likely contributed to the reduction in mast cell activation and to the beneficial effects of oral immunotherapy. These findings are consistent with previous studies implicating HRF in food allergy in humans. Collectively, these studies provide strong support for the concept that HRF-reactive IgEs contribute to intestinal inflammation in food-allergic patients and that the serum HRF-reactive IgE levels, not total or antigen-specific $\operatorname{IgE}$ levels, might be a predictor of successful immunotherapy in food-allergic patients.

\section{Concluding remarks}

Although the pursuit of HRF pathways as therapeutic targets for the treatment of allergic diseases may have finally come of age, much remains to be learned about the efficacy of HRF blockade in allergic disorders. For example, based on our current knowledge, it is unlikely that blockade of HRF-IgE interactions would modify allergic disease, but it may be effective at suppressing disease symptoms. The degree to which HRF inhibition may provide relief from established food-allergy symptoms is unclear, as it is not likely to block all IgE-dependent processes. Perhaps HRF blockade combined with traditional immunotherapy might be a better strategy, as HRF blockade would presumably provide protection against the occurrence of anaphylactic responses during immunotherapy regimes. Whether the benefits of $\mathrm{HRF}$ blockade will outweigh any potential side effects is unknown, especially as HRF plays such a critical role in several fundamental cellular processes, such as cell-cycle progression, proliferation, and survival. Although their results are not conclusive, Kawakami et al. (10) have demonstrated that blocking the IgE-binding sites on HRF does not seem to inhibit the effects of HRF on cellular growth or survival, suggesting that blockade of HRF's extracellular functions might be relatively safe. Despite these unknowns, these findings will undoubtedly fuel further clinical studies to determine whether HRF inhibition may prove to be a new tool in our arsenal to combat the ever-increasing morbidity of food allergy.

Address correspondence to: Marsha WillsKarp, Department of Environmental Health and Engineering, Johns Hopkins Bloomberg School of Public Health, 615 N. Wolfe St., RM W7527, Baltimore, Maryland 21205, USA. Phone: 410.955.2452; Email:mwkarp@jhu.edu.

1. Du Toit G, et al. Early consumption of peanuts in infancy is associated with a low prevalence of peanut allergy. JAllergy Clin Immunol.
2008;122(5):984-991.

2. Manohar M, Nadeau KC. The potential of antiIgE in food allergy therapy. Curr Treat Options Allergy. 2014;1(2):145-156.

3. Ando T, et al. Histamine-releasing factor enhances food allergy. J Clin Invest. 2017;127(12):4541-4553.

4. Alam R, Kuna P, Rozniecki J, Kuzminska B. The magnitude of the spontaneous production of histamine-releasing factor (HRF) by lymphocytes in vitro correlates with the state of bronchial hyperreactivity in patients with asthma. J Allergy Clin Immunol. 1987;79(1):103-108.

5. Sampson HA, Broadbent KR, BernhiselBroadbent J. Spontaneous release of histamine from basophils and histamine-releasing factor in patients with atopic dermatitis and food hypersensitivity. N Engl JMed. 1989;321(4):228-232.

6. Brunet C, Bedard PM, Lavoie A, Jobin M, Hebert J. Allergic rhinitis to ragweed pollen. II. Modulation of histamine-releasing factor production by specific immunotherapy. J Allergy Clin Immunol. 1992;89(1 pt 1):87-94.

7. Yenofsky R, Cereghini S, Krowczynska A, Brawerman G. Regulation of mRNA utilization in mouse erythroleukemia cells induced to differentiate by exposure to dimethyl sulfoxide. $\mathrm{Mol}$ Cell Biol. 1983;3(7):1197-1203.

8. Chitpatima ST, Makrides S, Bandyopadhyay R, Brawerman G. Nucleotide sequence of a major messenger RNA for a 21 kilodalton polypeptide that is under translational control in mouse tumor cells. Nucleic Acids Res. 1988;16(5):2350.

9. MacDonald SM, Rafnar T, Langdon J, Lichtenstein LM. Molecular identification of an IgE-dependent histamine-releasing factor. Science. 1995;269(5224):688-690.

10. Kawakami T, Kashiwakura J, Kawakami Y. Histamine-releasing factor and immunoglobulins in asthma and allergy. Allergy Asthma Immunol Res. 2014;6(1):6-12.

11. Schroeder JT, Lichtenstein LM, MacDonald SM. Recombinant histamine-releasing factor enhances IgE-dependent IL-4 and IL-13 secretion by human basophils. J Immunol. 1997;159(1):447-452.

12. Warner JA, Pienkowski MM, Plaut M, Norman PS, Lichtenstein LM. Identification of histamine releasing factor(s) in the late phase of cutaneous IgE-mediated reactions. J Immunol. 1986;136(7):2583-2587.

13. MacDonald SM, et al. Studies of IgE-dependent histamine releasing factors: heterogeneity of IgE. JImmunol. 1987;139(2):506-512.

14. Alam R, et al. Detection of histamine release inhibitory factor- and histamine releasing factor-like activities in bronchoalveolar lavage fluids. Am Rev Respir Dis. 1990;141(3):666-671.

15. Kuna P, Alam R, Kuzminska B, Rozniecki J. The effect of immunotherapy on the production of histamine-releasing factor (HRF) by mononuclear cells from patients with seasonal asthma: results of a double-blind, placebo-controlled, randomized study. J Allergy Clin Immunol. 1989;83(4):816-824.

16. Liao TN, Hsieh KH. Altered production of histamine-releasing factor (HRF) activity and 
responsiveness to $\mathrm{HRF}$ after immunotherapy in children with asthma. J Allergy Clin Immunol. 1990;86(6 pt 1):894-901.

17. Yeh YC, et al. The effects of overexpression of histamine releasing factor (HRF) in a transgenic mouse model. PLoS One. 2010;5(6):e11077.

18. Wantke F, MacGlashan DW, Langdon JM, MacDonald SM. The human recombinant histamine releasing factor: functional evidence that it does not bind to the IgE molecule. J Allergy Clin
Immunol. 1999;103(4):642-648.

19. Kashiwakura JC, et al. Histamine-releasing factor has a proinflammatory role in mouse models of asthma and allergy.J Clin Invest. 2012;122(1):218-228. 